\title{
Granulocyte-colony stimulating factor- and interleukin 6-producing diffuse deciduoid peritoneal mesothelioma
}

\author{
Noriko Kimura $^{1}$, Tetsuo Ogasawara ${ }^{2}$, Sho Asonuma ${ }^{2}$, Hikaru Hama ${ }^{3}$, Takashi Sawai ${ }^{4}$ \\ and Takayoshi Toyota ${ }^{2}$ \\ ${ }^{1}$ Department of Pathology and Laboratory Medicine; ${ }^{2}$ Department of Internal Medicine; ${ }^{3}$ Department of \\ Radiology, Tohoku Rosai Hospital, Sendai, Japan and ${ }^{4}$ Department of Pathology, Iwate Medical School, \\ Morioka, Japan
}

\begin{abstract}
An autopsy case of granulocyte colony-stimulating factor (G-CSF)- and interleukin-6 (IL-6)-producing diffuse deciduoid peritoneal mesothelioma is reported. The patient was a 70-year-old man with abdominal distension and weight loss in the year prior to his death. Laboratory data suggested severe inflammation with marked leukocytosis, thrombocytosis and elevated serum levels of C-reactive protein, G-CSF and IL-6. Imaging studies showed an expansive mass occupying the entire abdomen and pelvic cavity. Histological diagnosis of tissue taken by needle biopsy was difficult due to the unusual sarcomatoid-appearance of the tumor. In addition, there was severe infiltration of numerous neutrophilic leukocytes. An autopsy revealed that the diffuse peritoneal tumor had a fresh fishmeat-like appearance with focal mucinous degeneration and entirely encased the abdominal organs. Histological examination showed a sheet-like proliferation of tumor cells with large ovoid or polygonal cytoplasm, large atypical nuclei and obvious nucleoli. The tumor cells showed abundant glycogen and hyaluronic acid, and were immunoreactive to cytokeratin, calretinin, epithelial membrane antigen (EMA), CA-125, and focally to vimentin. The tumor cells were immunoreactive to G-CSF and IL-6. Electron microscopy revealed long, slender microvilli on the tumor cell surface. This tumor was diagnosed as a G-CSF- and IL-6producing, diffuse deciduoid mesothelioma. We report this case with special reference to the differential diagnosis of deciduoid peritoneal mesothelioma with paraneoplastic syndrome.
\end{abstract}

Modern Pathology (2005) 18, 446-450, advance online publication, 13 August 2004; doi:10.1038/modpathol.3800245

Keywords: deciduoid; mesothelioma; peritoneum; G-CSF; IL-6; immunohistochemistry; thrombocytosis

Diffuse peritoneal mesothelioma accounts for about a quarter of all cases of malignant mesothelioma. ${ }^{1}$ Nascimento et $a^{2}$ advocated a concept of deciduoid mesothelioma that includes its typical peritoneal location, a highly aggressive behavior with a poor prognosis and no etiological correlation with asbestos. The term 'deciduoid' was given due to the unusual histological pattern that closely resembles ectopic decidual reaction (deciduosis). Although the original publication reported that malignant deciduoid mesothelioma arises most commonly in the peritoneum of young female cases, recent

Correspondence: Dr N Kimura, MD, PhD, Department of Pathology and Laboratory Medicine, Tohoku Rosai Hospital, 21-3-4 Dainohara, Aoba-ku, Sendai 981-8563, Japan.

E-mail: nkimura-path@tohokuh.rofuku.go.jp

Received 04 March 2004; revised 29 June 2004; accepted 30 June 2004; published online 13 August 2004 publications revealed that it may also occur in the pleura or pericardium of elderly male and female subjects, with or without asbestos exposure. ${ }^{3-12}$ The pronounced anaplastic morphology of deciduoid mesothelioma requires differential diagnosis from other malignant tumors.

It is well known that leukocytosis and thrombocytosis are commonly encountered in a variety of inflammatory and neoplastic diseases. Thrombocytosis, defined by a platelet count above 400000 / $\mathrm{mm}^{3}$, has been almost as prevalent in diffuse malignant mesothelioma: $41 \%$ of patients, ${ }^{13} 83 \%{ }^{14}$ and $90 \%{ }^{15}$ had thromboembolic episodes. In addition, granulocytosis also has been previously reported in patients with mesothelioma. In this study, we present a patient with diffuse deciduoid peritoneal mesothelioma with special reference to the differential diagnosis by histology and paraneoplastic syndromes such as leukocytosis and thrombocytosis. 


\section{Materials and methods}

The case was an in-patient treated at the Tohoku Rosai Hospital, Sendai, Japan. Tissue specimens were fixed in $10 \%$ formalin, and $3 \mu \mathrm{m}$ sections were cut and then stained with hematoxylin and eosin. Periodic acid-Schiff staining followed by diastase digestion, and alcian blue staining followed by hyaluronidase digestion were performed on selected slides from the tumor. The immunohistochemical reagents used are shown in Table 1. Appropriate positive and negative controls were used in the staining process.

Electron microscopic examination was performed on an autopsy specimen fixed in a mixture of $2.5 \%$ glutaraldehyde and $2.0 \%$ paraformaldehyde buffered with $0.1 \mathrm{M}$ sodium cacodylate at $\mathrm{pH} 7.2$, and postfixed in $2 \%$ osmium tetroxide. Ultrathin sections stained with uranyl acetate and lead citrate were examined with a Hitachi 7100 transmission electron microscope (Hitachi 1000, Ltd, Tokyo, Japan).

Serum taken from the patient before death, was frozen at $-70^{\circ} \mathrm{C}$ until assayed. Serum levels of granulocyte colony-stimulating factor (G-CSF) and interleukin (IL-6) were measured commercially.

\section{Results}

\section{Case Report}

A 70-year-old man with abdominal distension and a 3-kg weight loss was admitted to a nearby hospital, where the accumulation of ascites without obvious tumor mass was noticed. After 3 months, he was transferred to the Rosai hospital for closer examination. Laboratory data revealed leukocytosis, throm-

Table 1 Antibodies and working dilutions

\begin{tabular}{lll}
\hline Antibodies & Sources & Dilutions \\
\hline $\begin{array}{l}\text { Cytokeratin } \\
\text { (KL1) }\end{array}$ & MBL, Nagoya, Japan & $1: 250$ \\
Vimentin & Boehringer Mannheim, Germany & $1: 15$ \\
Desmin & Bio-Science, Emmenbrucke, & $1: 1000$ \\
& Switzerland & \\
$\alpha$-SMA & DAKO, Glostrup, Denmark & $1: 500$ \\
Calretinin & ZYMED, San Francisco, CA, USA & $1: 10$ \\
CA125 & Novocastra, Newcastle upon Tyne, & $1: 100$ \\
& UK & $1: 2000$ \\
CA19-9 & Centocore, USA & $1: 200$ \\
Ki-67 & DAKO, Glostrup, Denmark & $1: 5000$ \\
CEA & MOCHIDA, Tokyo, Japan & $1: 5000$ \\
S100 & DAKO, Glostrup, Denmark & $1: 50$ \\
HMB45 & Enzo, NY, USA & $1: 100$ \\
EMA & DAKO, Glostrup, Denmark & $1: 1000$ \\
Myogenin & DAKO, Glostrup, Denmark & $1: 200$ \\
G-CSF (C-18) & Santa Cruz Biotech., Santa Cruz, & \\
& CA, USA & $1: 1200$ \\
IL-6 & Ohtsuka Pharm, Tokushima, Japan & \\
\hline
\end{tabular}

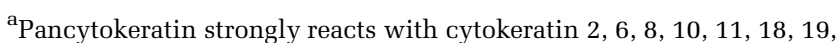
and weakly reacts with cytokeratin 5, and 14/15. bocytosis and elevated C-reactive protein (CRP) (Table 2). Computed tomography and magnetic resonance imaging detected a mass lesion occupying the entire abdomen and pelvic cavity. Gallium scintigraphy analysis showed dense staining throughout the abdomen, but not in the chest or bones (Figure 1). These imaging studies suggested either severe inflammatory or carcinomatous panperitonitis, diffuse peritoneal mesothelioma, or infiltration of some special type malignant lymphoma. The patient had no history of asbestos exposure.

Needle biopsy of the abdominal mass was carried out to make a diagnosis. The tissue taken was grossly gelatinous. Histological examination of the tissue revealed sheet-like atypical cells and large amount of mucinous substance in the background. The tumor cells had large and glassy eosinophilic cytoplasm, irregular-shaped nuclei and obvious nucleoli. Malignant tumors, such as metastasis of anaplastic large-cell carcinoma, rhabdomyosarcoma, malignant melanoma and malignant mesothelioma were suspected and included in the differential diagnosis. Glycogen was also detected in the tumor cells. A limited number of immunohistochemical stains was utilized due to a small biopsy specimen. The tumor cells were positive for cytokeratin, focally positive for vimentin and negative for CEA and CA19-9. These results suggested that the tumor was either carcinoma or mesothelioma, but not sarcoma. However, pronounced nuclear atypia of the tumor cells and the presence of large amounts of

Table 2 Laboratory data

\begin{tabular}{ll}
\hline Peripheral blood & \\
WBC & $46300 / \mathrm{mm}^{3}$ \\
Stab. & $17.5 \%$ \\
Seg. & $78.5 \%$ \\
Monocytes & $2.5 \%$ \\
Lymphocytes & $1.5 \%$ \\
RBC & $437 \times 10^{4} / \mathrm{mm}^{3}$ \\
Hb & $11.9 \mathrm{~g} / \mathrm{dl}$ \\
Ht. & $37.2 \%$ \\
Platelet & $85 \times 10^{4} / \mathrm{mm}^{3}$ \\
& \\
Biochemistry & \\
ALP & $1513 \mathrm{IU} / \mathrm{l}$ \\
LDH & $331 \mathrm{IU} / \mathrm{ml}$ \\
TP & $6.9 \mathrm{~g} / \mathrm{dl}$ \\
Alb & $2.4 \mathrm{~g} / \mathrm{dl}$ \\
BUN & $39 \mathrm{mg} / \mathrm{dl}$ \\
Cre & $0.75 \mathrm{mg} / \mathrm{dl}$ \\
CRP & $28 \mathrm{mg} / \mathrm{dl}$ \\
& \\
Tumor markers & \\
CEA & $3.3 \mathrm{ng} / \mathrm{ml}(5.0>)$ \\
CA19-9 & $37 \mathrm{U} / \mathrm{ml}(37>)$ \\
CA125 & $971 \mathrm{U} / \mathrm{ml}(220-530)$ \\
G-CSF & $147 \mathrm{pg} / \mathrm{ml}(4.0>)$ \\
IL-6 & $317 \mathrm{U} / \mathrm{ml}(35>)$ \\
Hyarulonic acid & $345 \mathrm{ng} / \mathrm{ml}(50>)$ \\
Thromobomodullin & $5.4 \mathrm{FU} / \mathrm{ml}(2.1-4.1)$ \\
SCC & $0.5 \mathrm{ng} / \mathrm{ml}(1.5>)$ \\
CYFRA & $11 \mathrm{ng} / \mathrm{ml}(3.5>)$ \\
\hline
\end{tabular}




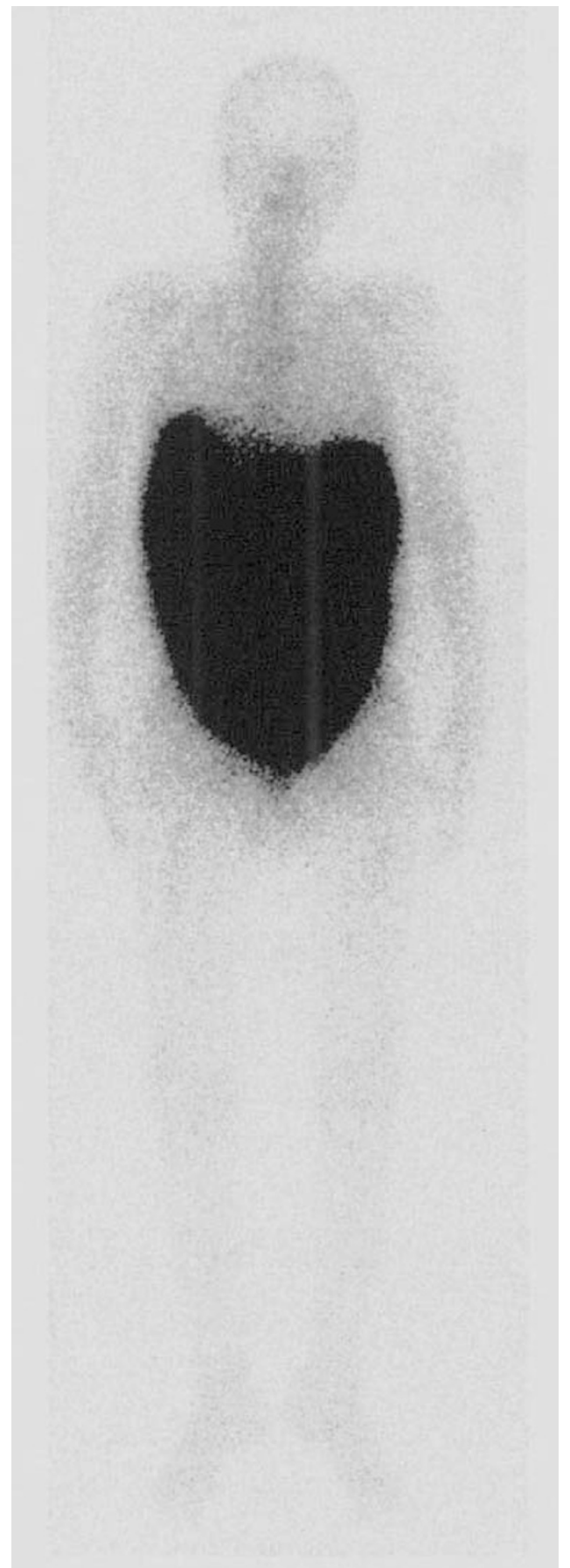

Figure 1 Gallium scintigraphy shows dense staining throughout the abdomen and pelvic cavity but not in the nearby chest or bones.

extracellular mucinous substance were unusual for an ordinary diffuse mesothelioma. A definite diagnosis of peritoneal mesothelioma was made difficult by the limited size of the biopsy specimen. The patient deteriorated day by day and died of cachexia 2 weeks after biopsy and 1 year after clinical onset.

An autopsy was performed $1 \mathrm{~h}$ after death. The abdominal and pelvic cavities were entirely occupied by the tumor tissue, which showed gelatinous, whitish and fresh fishmeat-like mass. The abdominal organs, including the intestinal loops, liver, gall bladder and spleen were encased by the tumor and were unable to separate from each other due to severe adhesion (Figure 2). In contrast, kidneys, pleural cavity and lungs were unaffected. Histology of the tumor tissue showed that the tumor cells were arranged in a sheet-like pattern. Mucinous degeneration was focally observed. The tumor cells had abundant eosinophilic cytoplasm with irregularshaped large nuclei and obvious nucleoli. Severe infiltration of neutrophilic leukocytes around the tumor cells was evident (Figure 3). Nuclear atypia was evident. Multinucleated giant cells were occasionally seen. Both glycogen, detected by digestion with diastase, and hyaluronic acid, digested with hyaluronidase, were present. The mucinous substance, also treated with hyaluronidase, digested incompletely.

Immunohistochemical staining of the tumor was positive for cytokeratin, calretinin (Figure 4), epithelial membrane antigen (EMA), CA-125 and vimentin focally, but negative for $\alpha$-smooth muscle actin $(\alpha-$ SMA), desmin, S100, HMB-45 and myogenin. G-CSF (Figure 5) and IL-6 (Figure 6) immunoreactivity was also clearly observed in the tumor cells. Immunoreactivity to Ki-67 was seen in about $10 \%$ of the cells in the solid area, and not seen in mucinous area. Electron microscopy revealed long, slender microvilli on the tumor surface circumferentially, and bundles of intermediate-sized filaments in the cytoplasm.

Measurements of G-CSF and IL-6 in the serum were $147 \mathrm{pg} / \mathrm{ml}$ (normal range: $4.0 \mathrm{pg} / \mathrm{ml}>$ ) and $317 \mathrm{U} / \mathrm{ml}$ (normal range: $35.0 \mathrm{U} / \mathrm{ml}>$ ), respectively. All of these findings were consistent with G-CSFand IL-6-producing deciduoid diffuse peritoneal mesothelioma.

\section{Discussion}

Diffuse deciduoid mesothelioma is an extremely rare tumor, and only about 30 cases have been reported. ${ }^{16}$ The cases have been reported from the viewpoint of the differential diagnosis of metastasis of anaplastic adenocarcinomas, malignant melanoma, rhabdomyosarcoma and other unspecified sarcomas involving serosal membranes. Deciduoid mesothelioma is distinguished from other tumors by positive staining for cytokeratin, vimentin, calretinin, HMBE-1, EMA, CA125 and thrombomodulin and negative staining for CEA, HMB45 and myogenin. ${ }^{17-20}$ Some cases showed rhabdoid changes in deciduoid mesothelioma. ${ }^{9}$

The presence of intracytoplasmic glycogen and hyaluronic acid also are important evidences for diagnosis of mesothelioma. Long slender microvilli observed with electron microscopy are also a characteristic for mesothelioma. 


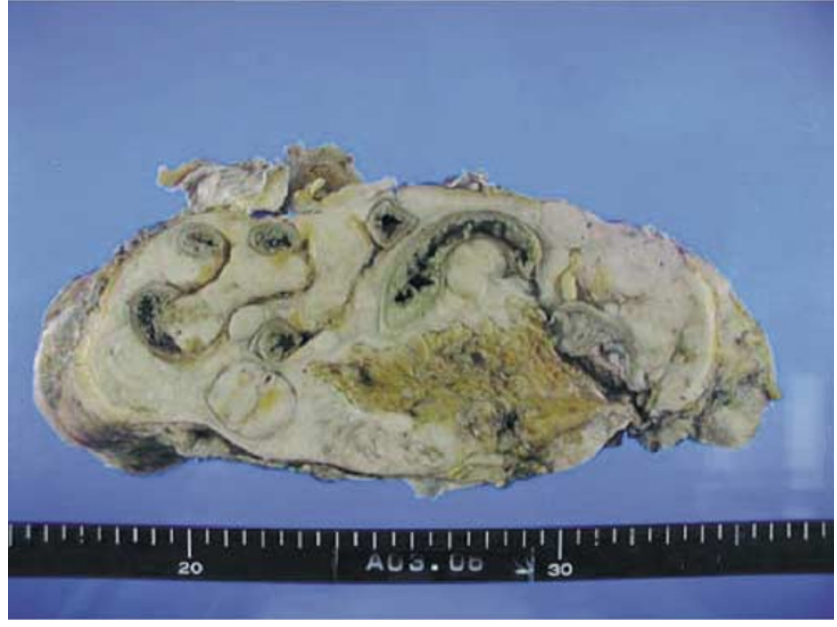

Figure 2 A view of cross-section of the abdominal mass. Abdominal organs such as the intestinal loops, liver and spleen are encased by the tumor (autopsy specimen).

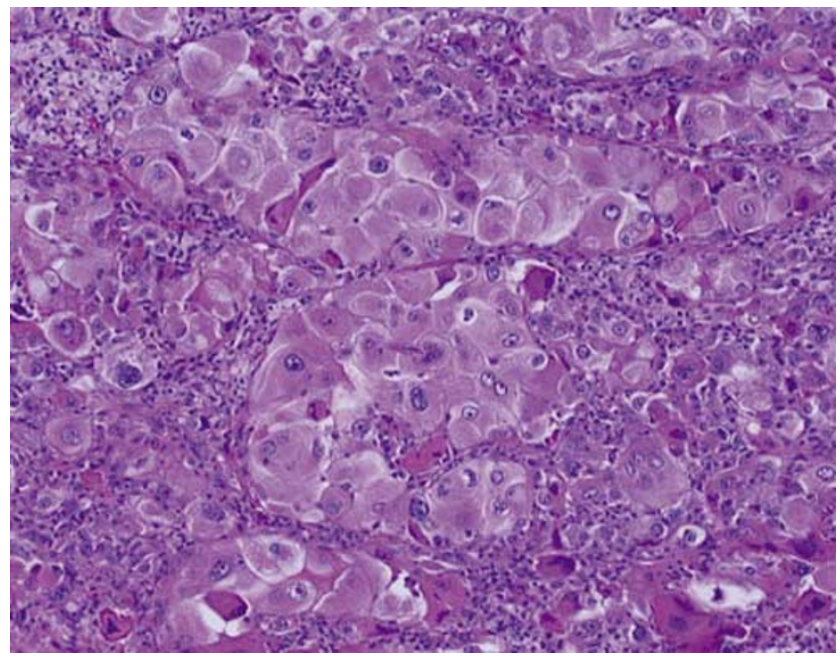

Figure 3 Sheet-like arrangement of the tumor cells showing abundant eosinophilic cytoplasm, irregular-shaped large nuclei and obvious nucleoli. Multinucleated giant cells are occasionally seen. Severe infiltration of neutrophilic leukocytes to the tumor cells is characteristically seen.

Additionally, a high prevalence of thrombocytosis in malignant mesothelioma has been reported. The incidence of thrombocytosis was $83 \%$ in patients with malignant mesothelioma, and, in contrast, $7.5 \%$ in patients with lung carcinoma. ${ }^{14}$ Higashihara et $a l^{21}$ reported a patient with malignant peritoneal mesothelioma showing marked thrombocytosis, and producing large amounts of IL-6 and small amounts of granulocyte-macrophage colony-stimulating factor (GM-CSF) and monocyte colony-stimulating factor (M-CSF). Schmitter et $a l^{22}$ established seven human malignant pleural mesothelioma cell lines, and revealed that all mesothelioma cell lines continuously produce IL-6; however, G-CSF production was detected in only one cell line. Since IL-6

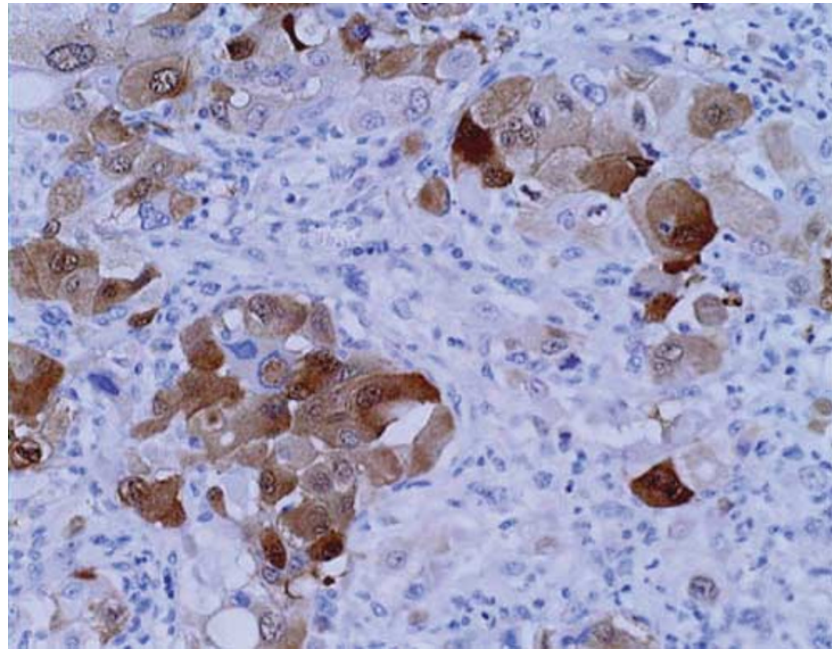

Figure 4 Calretinin is recognized in both the nuclei and cytoplasm of the tumor cells.

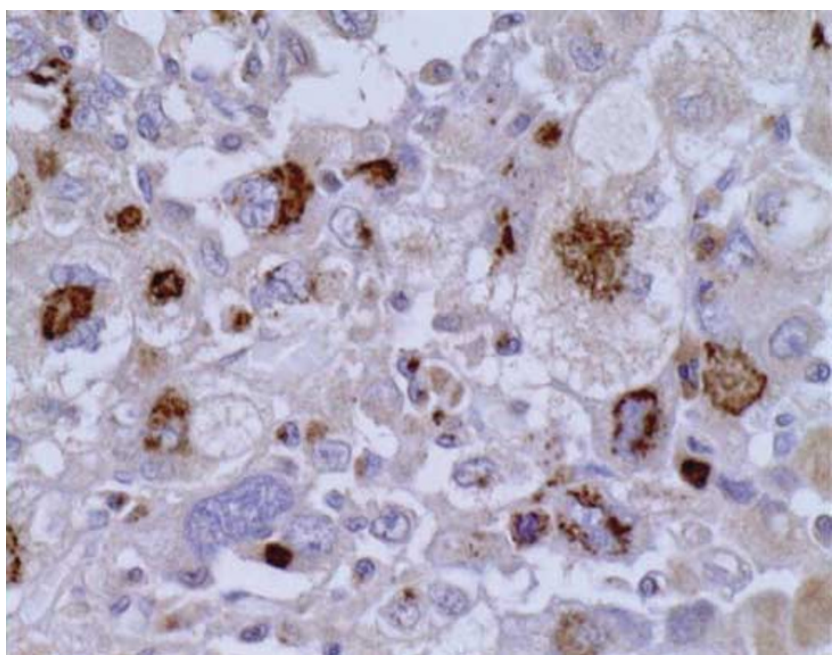

Figure 5 G-CSF is strongly and granularly stained in the cytoplasm.

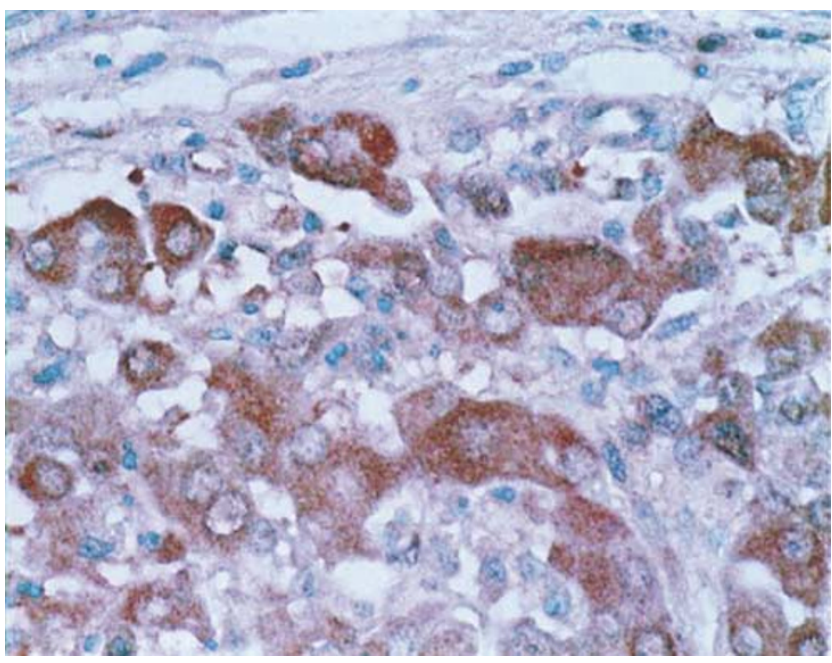

Figure 6 IL-6 is diffusely stained in the cytoplasm of the tumor cells. 
induces thrombocytosis by stimulating thrombopoietin, ${ }^{23}$ persistent secretion of IL-6 seemed to promote thrombocytosis in the present patient. A small number of G-CSF-producing mesotheliomas have been previously reported in a pleural mesothelioma, ${ }^{24,25}$ and a pericardial mesothelioma, ${ }^{4}$ but not in decidual mesothelioma. However, severe inflammatory cell infiltration is commonly observed in the tumor tissues of the cases of deciduoid mesothelioma reported previously. ${ }^{1,2,17,19}$ The incidence of G-CSF or GM-CSF-producing mesothelioma may be higher than that reported. In addition to immunohistochemistry or measurement of serum levels, mRNA detection by RT-PCR may be used to confirm the production of G-CSF in tumor tissue or in the primary culture of tumor tissue. ${ }^{4}$

The data showing such elevated cytokines may lead clinicians to diagnose inflammatory disease rather than neoplastic disease. It is important to recognize this paraneoplastic phenomenon associated with some mesotheliomas including deciduoid mesothelioma.

\section{Acknowledgements}

We thank Mr S Sugaki, Mr K Takashima and Ms $\mathrm{K}$ Abe for their technical assistance.

\section{References}

1 Battifora H, McCaughey WTE. Diffuse malignant mesothelioma. In: Battifora H, McCaughey (eds). Tumors of the Serosal Membranes. Atlas of Tumor Pathology. AFIP: Washington, DC, 1995, pp 17-88.

2 Nascimento AG, Keeney GL, Fletcher CD. Deciduoid peritoneal mesothelioma. An unusual phenotype affecting young females. Am J Surg Pathol 1994;18: 439-445.

3 Gloeckner-Hofmann $\mathrm{K}$, Zhu XZ, Bartels $\mathrm{H}$, et al. Deciduoid pleural mesothelioma affecting a young female without prior asbestos exposure. Respiration 2000;67:456-458.

4 Horio H, Nomori H, Morinaga S, et al. Granulocyte colony-stimulating factor-producing primary pericardial mesothelioma. Human Pathol 1999;30:718-720.

5 Monaghan H, Al-Nafussi A. Deciduoid pleural mesothelioma. Histopathology 2001;39:104-106.

6 Okonkwo A, Musunuri S, Diaz Jr L, et al. Deciduoid mesothelioma: a rare, distinct entity with unusual features. Ann Diagn Pathol 2001;5:168-171.

7 Ordonez NG. Epithelial mesothelioma with deciduoid features: report of four cases. Am J Surg Pathol 2000;24:816-823.

8 Orosz Z, Nagy P, Szentirmay Z, et al. Epithelial mesothelioma with deciduoid features. Virchows Arch 1999;434:263-266.
9 Puttagunta L, Vriend RA, Nguyen GK. Deciduoid epithelial mesothelioma of the pleura with focal rhabdoid change. Am J Surg Pathol 2000;24: 1440-1443.

10 Reis-Filho JS, Pope LZ, Milanezi F, et al. Primary epithelial malignant mesothelioma of the pericardium with deciduoid features: cytohistologic and immunohistochemical study. Diagn Cytopathol 2002;26: 117-122.

11 Shia J, Erlandson RA, Klimstra DS. Deciduoid mesothelioma: a report of 5 cases and literature review. Ultrastruct Pathol 2002;26:355-363.

12 Talerman A, Chilcote RR, Montero JR, et al. Diffuse malignant peritoneal mesothelioma in a 13-year old girl. Am J Surg Pathol 1985;9:73-80.

13 Ruffie P, Feld R, Minkin S, et al. Diffuse malignant mesothelioma of the pleura in Ontario and Quebec: a retrospective study of 332 patients. J Clin Oncol 1989;7:1157-1168.

14 Nakano T, Fujii J, Tamura S, et al. Thrombocytosis in patients with malignant pleural mesothelioma. Cancer 1986;58:1699-1701.

15 Chahinian AP, Pajak TF, Holland JF, et al. Diffuse malignant mesothelioma. Prospective evaluation of 69 patients. Ann Intern Med 1982;96:746-755.

16 Maeda S, Hosone M, Katayama H, et al. Deciduoid mesothelioma in the pelvic cavity. Pathol Int 2004;54:67-72.

17 Chikai K, Ota S, Makino Y, et al. A case of deciduoid mesothelioma. Jpn J Diagn Pathol 2003;20:270-272 (in Japanese).

18 Serio G, Scattone A, Pennella A, et al. Malignant deciduoid mesothelioma of the pleura: report of two cases with long survival. Histopathology 2002;40: 348-352.

19 Shanks JH, Harris M, Banerjee SS, et al. Mesotheliomas with deciduoid morphology: a morphologic spectrum and a variant not confined to young females. Am J Surg Pathol 2000;24:285-294.

20 Urabe S, Tsuji K, Koba F, et al. A case of peritoneal mesothelioma arising from greater omentum. Jpn J Diagn Pathol 2003;20:146-149 (in Japanese).

21 Higashihara $\mathrm{M}$, Sunaga $\mathrm{S}$, Tange $\mathrm{T}$, et al. Increased secretion of interleukin-6 in malignant mesothelioma cells from a patient with marked thrombocytosis. Cancer 1992;70:2105-2108.

22 Schmitter D, Lauber B, Fago B, et al. Hematopoietic growth factors secreted by seven human pleural mesothelioma cell lines: interleukin-6 production as a common feature. Int J Cancer 1992;51:296-301.

23 Kaser A, Brandacher G, Steurer W, et al. Interleukin-6 stimulates thrombopoiesis through thrombopoietin: role in inflammatory thrombocytosis. Blood 2001; 98:2720-2725.

24 KasugaI, Ishizuka S, Minemura K, et al. Malignant pleural mesothelioma produces functional granulocyte-colony stimulating factor. Chest 2001;119: 981-983.

25 Rikimaru T, Ichikawa Y, Ogawa Y. Production of granulocyte colony-stimulating factor (G-CSF) by malignant mesothelioma. Eur Respir J 1995;8:183-184. 